Sofern Schulreformen einen Schwerpunkt auf Bildungsgerechtigkeit (im Gegensatz zu Bildungsqualität) legen, haben sie in gewisser Weise umverteilenden Charakter. Während der potenzielle Verlierer solcher Reformen, das Bildungsbürgertum, als Ingroup gut aktivierbar und organisierbar ist, gilt für die potentiellen Gewinner einer solchen Reform - sozial Benachteiligte und/oder Menschen mit Migrationshintergrund - als Outgroups das Gegenteil. Vor diesem Hintergrund erscheint es wahrscheinlich, dass die „natürlichen“ Gegner einer solchen umverteilenden Reform direktdemokratische Verfahren nutzen, um ihre Interessen durchzusetzen, wobei vergleichsweise niedrige Quoren von Vorteil sind. Gegen die Veränderung des Status quo ist es zudem tendenziell leichter, breitere Unterstützung zu finden, als für einen Policy-Wandel. Dass die Nutzung dieser Verfahren im Hamburger Fall so überaus erfolgreich war, wurde allerdings von zwei Faktoren befördert, die in anderen Fällen möglicherweise nicht gegeben sind: Erstens diente das direktdemokratische Verfahren in gewissem Maße der Kompensation der Willensbildung innerhalb der Hamburger CDU sowie zwischen der CDU und ihrer Wählerschaft, die durch die mächtige Stellung Ole von Beusts und dessen Bekenntnis zur Primarschule verstellt war. Zweitens resultiert die ganz breite, sicher über das Bildungsbürgertum hinausgehende Ablehnung der Schulreform durch viele Hamburger in erster Linie aus der - taktisch unklugen - Abschaffung des (schon zuvor nicht unbegrenzten) Elternwahlrechts. Damit bekam die Reform den Nimbus einer links-autoritären Intervention in die Schulbildung und auch in die Bildungsautonomie der Familien, der ihr selbst dann noch anhaftete, als das Elternwahlrecht bereits wiederhergestellt war - bei der Volksabstimmung.

Abschließend kann man also für die weitere empirische Überprüfung die Hypothese aufstellen, dass Schulreformen mit Fokus auf Chancengleichheit aufgrund der enthaltenen Umverteilungswirkung bei ungleicher Handlungsfähigkeit der von der Reform Begünstigten (Outgroups) und der Belasteten (Ingroups) durch direktdemokratische Verfahren mit niedrigen Quoren mit einiger Wahrscheinlichkeit verhindert werden.

\title{
E-Petitionen in Deutschland: Zwischen niedrigschwelligem Partizipationsangebot und quasi-plebiszitärer Nutzung
}

\author{
Andreas Jungherr und Pascal Jürgens*
}

Seit der Einführung der E-Petitionsplattform des Deutschen Bundestags im Jahr 2005 haben einzelne E-Petitionen immer wieder hohe Unterstützerzahlen gefunden und damit Medieninteresse und öffentliche Aufmerksamkeit erregt - sei es eine E-Petition für die Einführung eines bedingungslosen Grundeinkommens ${ }^{1}$, gegen die Sperrung von Internetseiten im

* Die Autoren möchten Harald Schoen, Helen Margetts, Tobias Escher, Ralf Lindner, Frank Bergmann, Thomas Kling und Joachim Jungherr für hilfreiche Diskussionen über den Untersuchungsgegenstand danken.

1 Vgl. Susanne Wiest, Petition: Reformvorschläge in der Sozialversicherung - Bedingungsloses Grundeinkommen vom 10. Dezember 2008, Deutscher Bundestag, https://epetitionen.bundestag.de/index.php?action=petition;sa=details;petition=1422 (Abruf am 18. Februar 2011). 
Kontext der Diskussion um das Zugangserschwerungsgesetz ${ }^{2}$, zur Unterstützung freiberuflicher Hebammen ${ }^{3}$ oder gegen den Atomkompromiss von 2010. ${ }^{4}$ Bei der Berichterstattung über E-Petitionen fällt auf, dass Journalisten der Mobilisierung von Unterstützern durch die Petenten ähnlich viel Aufmerksamkeit widmen wie dem eigentlichen thematischen Anliegen der Petition. Kommentatoren sprechen von einer „neue[n] Protestform“ für „die Internet-Community“. Petitionen stellen „Rekorde“ auf oder werden zu „Hits“5, sie legen „sensationelle Aufholjagd[en]"6 hin, oder ihre „Unterstützer klicken im Sekundentakt" Formulierungen dieser Art sind aus der „Horse-Race“-Berichterstattung über Wahlkämpfe bekannt, in der Journalisten zunehmend über Veränderungen in Meinungsumfragen anstatt über thematische Aspekte des Wahlkampfs sprechen. ${ }^{8}$ Ähnliches ist angesichts der teils sprunghaft ansteigenden und stets gut dokumentierten Unterstützerzahlen auf der E-Petitionsplattform festzustellen.

Ein weiteres Element vieler Berichte über erfolgreiche E-Petitionen ist die Konzentration auf die Person, die eine öffentliche E-Petition formulierte und auf der Petitionsplattform des Deutschen Bundestags einstellte. ${ }^{9}$ Die Initiatoren werden so zu inoffiziellen Sprechern

2 Vgl. Franziska Heine, Petition: Internet - Keine Indizierung und Sperrung von Internetseiten vom 22. April 2009, Deutscher Bundestag, https://epetitionen.bundestag.de/index.php?action=p etition;sa=details;petition=3860 (Abruf am 18. Februar 2011).

3 Vgl. Martina Klenk, Petition: Heilhilfsberufe - Sofortmaßnahmen zur wohnortnahen Versorgung mit Hebammenhilfe vom 14. April 2010, Deutscher Bundestag, https://epetitionen.bundestag. de/index.php?action=petition;sa=details;petition=11400 (Abruf am 18. Februar 2011).

4 Vgl. Jörg Zwosta, Petition: Nukleare Ver- und Entsorgung - Einhaltung der Verträge zur Abschaltung der Atomkraftwerke bis zum Jahr 2023 vom 17. August 2010, Deutscher Bundestag, https://epetitionen.bundestag.de/index.php?action=petition;sa=details;petition=13587. Vgl. exemplarisch zum Grundeinkommen: Thomas Vitzhum, 50.000 Stimmen für Grundeinkommen für alle, in: Welt Online vom 18. Februar 2009, http://www.welt.de/politik/article3228781/50-000Stimmen-fuer-Grundeinkommen-fuer-alle.html. Zu Netzsperren: Christian Rath, Die OnlineOpposition, in: taz.de vom 8. August 2009, http://www.taz.de/1/netz/artikel/1/die-online-opposition/. Zu Hebammen: Simone Schmollack, Hebammen starten Online-Petition, in: taz.de vom 19. Mai 2010, http://www.taz.de/1/politik/deutschland/artikel/1/hebammen-starten-online-petition/. Zum Atomkompromiss: Martin Motzkau, Rückschlag für das Zukunftsmodell, in: stern.de vom 28. Oktober 2010, http://www.stern.de/politik/deutschland/online-petition-zu-atomkraftrueckschlag-fuer-das-zukunftsmodell-1617744.html (Abruf aller Websites am 18. Februar 2011).

5 Vorangehende Zitate aus Ulf Schönert, Web statt Fußgängerzone, in: stern.de vom 17. Juli 2009, http://www.stern.de/digital/online/online-petitionen-web-statt-fussgaengerzone-706561.html (Abruf am 18. Februar 2011).

6 Martin Motzkau, a.a.O. (Fn. 4).

7 tagesschau.de, Hebammen-Unterstützer klicken im Sekundentakt, vom 11. Mai 2010, http:// www.tagesschau.de/inland/hebammen 110.html (Abruf am 18. Februar 2011).

8 Vgl. Julianne F. Flowers / Audrey A. Haynes / Michael H. Crespin, The Media, the Campaign, and the Message, in: American Journal of Political Science, 47. Jg. (2003), H. 2, S. 259 - 273; Udo Michael Krüger / Thomas Zapf-Schramm, Wahlinformationen im öffentlich-rechtlichen und privaten Fernsehen 2009, in: Media Perspektiven 12/2009, S. 622 - 636.

9 So begegnen wir in der Berichterstattung zu E-Petitionen Susanne Wiest, Initiatorin der E-Petition für ein bedingungsloses Grundeinkommen, Franziska Heine, Initiatorin der E-Petition gegen Onlinesperren, und Jörg Zwosta, Initiator der E-Petition gegen den Atomkompromiss. Vgl. Waltraud Schwab, Probiert doch mal das, in: taz.de vom 10. Februar 2009, http://www.taz.de/1/leben/koepfe/artikel/1/probiert-doch-mal-das/ (Abruf am 18. Februar 2011); Thomas Knüwer, Es mischen sich jetzt Online- und reale Welt, in: Handelsblatt vom 8. Mai 2009, http://www.handelsblatt.com/technologie/it-tk/it-internet/es-mischen-sich-jetzt-online-und-reale-welt/3172778. html (Abruf am 18. Februar 2011); Martin Motzkau, a.a.O. (Fn. 4). 
der von ihrer Petition aufgegriffenen politischen Themen. Besonders deutlich wird dies im Fall von Franziska Heine, die im Frühjahr 2009 für viele zum Gesicht der „Netzgemeinde“ wurde. Exemplarisch zeigt sich dies an einem von der Wochenzeitung Die Zeit organisierten Gespräch zwischen der damaligen Familienministerin Ursula von der Leyen, verantwortlich für das so genannte Gesetz zur Erschwerung des Zugangs zu kinderpornographischen Inhalten in Kommunikationsnetzen, und Franziska Heine, Initiatorin der E-Petition gegen Netzsperren und damit Vertreterin der Internetaktivisten. ${ }^{10}$

Diese beiden Charakteristiken, die eindeutig dokumentierte Entwicklung von Unterstützern von politischen Themen und die intuitive Personalisierung dieser Themen, machen E-Petitionen zu einem sehr medienfreundlichen E-Government-Element. Dies führt dazu, dass ihnen durch Kommentatoren immer wieder quasi-plebiszitäre Funktionen zugeschrieben werden. Sie seien „eine Form von direkter Demokratie“11, „Machtinstrument" der „Online-Opposition“12 oder Ausdruck des „Aufstand[s] im Netz“13. Es entsteht also der Eindruck, dass E-Petitionen eine Form direktdemokratischer Willensbildung seien. Auch wenn das Sammeln von Unterstützern auf den ersten Blick den Abstimmungen genuin direktdemokratischer Instrumente, zum Beispiel Volksentscheiden, ähneln mag, so ist selbst erfolgreichen E-Petitionen lediglich eine öffentliche Anhörung des Petenten vor dem Petitionsausschuss garantiert.

Der Eindruck, es handele sich um direktdemokratische Instrumente, kommt allerdings politischen Akteuren entgegen. Zunehmend nutzen Parteien und Verbände die E-Petitionsplattform des Bundestags, um Unterstützung für ihre politischen Vorhaben medienwirksam sichtbar zu machen. So reichte zum Beispiel die Präsidentin des Deutschen Hebammenverbandes Martina Klenk die E-Petition „Heilhilfsberufe - Sofortmaßnahmen zur wohnortnahen Versorgung mit Hebammenhilfe" ein und erreichte im Laufe der Mitzeichnungsfrist 105.386 Unterschriften. ${ }^{14}$ Politische Akteure nutzen jedoch auch indirekte Wege, um durch hohe Mitzeichnerzahlen Unterstützung für ihre politischen Anliegen zu finden. So warb die Partei Bündnis 90/Die Grünen im Oktober 2010 auf ihrer Internetseite ${ }^{15}$ und ihrem Twitter-Feed ${ }^{16}$ für die Mitzeichnung der E-Petition „Nukleare Ver- und Entsorgung - Einhaltung der Verträge zur Abschaltung der Atomkraftwerke“, um nach Abschluss der Zeichnungsfrist die hohen Unterstützerzahlen als stellvertretend für Mehrheitsverhältnisse in der Bevölkerung zu erklären. ${ }^{17}$

10 Vgl. Kai Biermann / Heinrich Wefing, Ihnen ist egal, was wir denken, in: Zeit Online vom 1. September 2009, http://www.zeit.de/online/2009/26/leyen-heine-netzsperren (Abruf am 18. Februar 2011).

11 Thomas Vitzhum, a.a.O. (Fn. 4).

12 Christian Rath, a.a.O. (Fn. 4).

13 Martin Motzkau, a.a.O. (Fn. 4).

14 Vgl. Martina Klenk, a.a.O. (Fn. 3).

15 Vgl. Bündnis 90/Die Grünen, Bundestagspetition gegen Atomkraft knackt die 50.000, 22. Oktober 2010, http://www.gruene-bundestag.de/cms/atomausstieg/dok/358/358029.abstimmen_ gegen_akwlaufzeitverlaengerung.html (Abruf am 22. Oktober 2010).

16 Vgl. @Die_Gruenen, EILT! BT-Petition gg Atomkraft!, 21. Oktober 2010, http://twitter.com/ Die_Gruenen/status/28035523285 (Abruf am 18. Februar 2011).

17 Exemplarisch Sylvia Kotting-Uhl/ Memet Kilic, Bürger gegen Laufzeitverlängerungen, Bündnis 90 Die Grünen Bundestagsfraktion, 22. Oktober 2010, http://www.gruene-bundestag.de/cms/presse/dok/358/358050.buerger_gegen_laufzeitverlaengerungen.html (Abruf am 18. Februar 2011). 
E-Petitionen sind also schon längst mehr als die „Notrufsäule des kleinen Mannes“18. Es ist folglich notwendig, zu einem besseren Verständnis der Nutzungsdynamiken der E-Petitionsplattform des Bundestags zu gelangen. Wie RalfLinder und Ulrich Riehm 2009 feststellten, ist die Literatur zum deutschen Petitionswesen verhältnismäßig überschaubar. ${ }^{19}$ Seitdem hat das Büro für Technikfolgen-Abschätzung beim Deutschen Bundestag (TAB) durch die Veröffentlichungen von Ergebnissen des Projekts „Öffentliche elektronische Petitionen und bürgerschaftliche Teilhabe" umfangreiche Grundlagenarbeit zu diesem Thema geleistet. ${ }^{20}$ Diese Arbeiten sollen hier aus einer neuen Forschungsperspektive ergänzt werden.

In den letzten Jahren hat sich durch die starke Verbesserung der Rechenleistung von Computern und der immer weiter verbreiteten Nutzung unterschiedlicher internetbasierter Online-Dienste ein Ansatz herausgebildet, der versucht, durch die computergestützte Analyse von Daten, die bei der Nutzung von Internet-Plattformen entstehen, Erkenntnisse über menschliches Verhalten zu gewinnen. Dieser Forschungsansatz wird mit den Begriffen "Computational Social Science“21 und „Digitale Methoden“22 umschrieben. Die E-Petitionsplattform des Bundestags bietet Forschern eine interessante Datengrundlage und im Vergleich zu standardmäßigen Untersuchungsmethoden, wie zum Beispiel Umfragen oder Interviews mit Petenten, neue Erkenntnismöglichkeiten. Wie verhalten sich Nutzer auf der Plattform? Zeichnen sie eine Petition oder viele? Welche E-Petitionen sind erfolgreich? Welche teilen Mitzeichner und sind also durch ihre Unterstützer verbunden? Die Antworten auf diese Fragen führen zu einem besseren Verständnis der Plattform als Ganzes und der Erfolge einzelner E-Petitionen. ${ }^{23}$

\section{E-Petitionen an den Deutschen Bundestag}

In Artikel 17 GG ist ein allgemeines Petitionsrecht verankert, demgemäß sich Bürger mit schriftlich eingereichten Bitten oder Beschwerden an eine dafür zuständige Behörde wenden können. ${ }^{24}$ Im Fall von Petitionen an den Bundestag werden diese durch den Petitions-

18 RalfLindner / Ulrich Riehm, Modernisierung des Petitionswesens und der Einsatz neuer Medien, in: ZParl, 40. Jg. (2009), H. 3, S. 495 - 512, S. 496.

19 Vgl. ebenda, S. 495.

20 Vgl. Ulrich Riehm / Christopher Coenen / RalfLindner / Clemens Blümel, Bürgerbeteiligung durch E-Petitionen: Analysen von Kontinuität und Wandel im Petitionswesen, Berlin 2009; Ralf Lindner / Ulrich Riehm, a.a.O. (Fn. 18), S. 495 - 512; Ulrich Riehm / Matthias Trénel, Öffentliche Petitionen beim Deutschen Bundestag: Ergebnisse einer Petentenbefragung, in: ZParl, 40. Jg. (2009), H. 3, S. 512 - 528; Ulrich Riehm / Christopher Coenen / RalfLindner, Zur Öffentlichkeit des Petitionsverfahrens beim Deutschen Bundestag und beim Schottischen Parlament, in: ZParl, 40. Jg. (2009), H. 3, S. $529-543$.

21 David Lazer / Alex Pentland / Lada Adamic / Sinan Aral / Albert-László Barabási / Devon Brewer / Nicholas Christakis / Noshir Contractor / James Fowler / Myron Gutmann / Tony Jebara / Gary King / Michael Macy / Deb Roy / Marshall Van Alstyne, Computational Social Science, in: Science, 129. Jg. (2009), H. 5915, S. $721-723$.

22 Richard Rogers, The End of the Virtual: Digital Methods, Amsterdam 2009.

23 Für eine ausführliche Diskussion des Forschungsansatz vgl. Andreas Jungherr / Pascal Jürgens, The Political Click: Political Participation through E-Petitions in Germany, in: Policy \& Internet, 2. Jg. (2010), H. 2, S. $131-165$.

24 Vgl. Art. 17 GG. 
ausschuss bearbeitet. Im September 2005 startete das Parlament ein Pilotprojekt, das es Petenten möglich machte, ihre Petition über eine elektronische Plattform einzureichen. ${ }^{25}$ Im Oktober 2008 wurde diese nach Überarbeitung in den regulären Betrieb übernom$m^{26}{ }^{26}$, so dass seither auf diesem Wege Einzelpetitionen eingereicht werden können. Dies sind so genannte Online-Petitionen, die sich nur auf die spezifische Situation des Petenten beziehen und von diesem nicht zur allgemeinen Mitzeichnung gedacht wurden. Petenten haben außerdem die Möglichkeit, auf der E-Partizipationsplattform eine „Öffentliche Petition" einzustellen und damit anderen Nutzern die Möglichkeit zu geben, diese mitzuzeichnen. ${ }^{27}$ Die folgenden Analysen beziehen sich ausschließlich auf diese Öffentlichen Petitionen. Nach einer formalen Prüfung durch den Petitionsausschuss werden sie auf der E-Plattform veröffentlicht. Daraufhin haben Nutzer sechs Wochen Zeit, sie mitzuzeichnen. Nach dieser Frist wird die Petition unabhängig von der Anzahl ihrer Zeichner durch den Petitionsausschuss bearbeitet. Gelingt es einer „Öffentlichen Petition“ jedoch innerhalb der ersten drei Wochen ihrer Mitzeichnungsfrist über 50.000 Unterschriften zu gewinnen, ist der Petitionsausschuss dazu verpflichtet, den Petenten für eine Anhörung in eine öffentliche Ausschusssitzung einzuladen. ${ }^{28}$ Es überrascht wenig, dass diese 50.000 Mitzeichner inzwischen zu einem Meilenstein in jeder Kampagne für E-Petitionen geworden sind. Man liest von einer „magischen Grenze“ 29 , die „durchbrochen “30 oder „geknackt" ${ }^{31}$ wird. Unterstützerseiten einzelner E-Petitionen werben ganz gezielt für das Erreichen dieser Zahl ${ }^{32}$ - und das nicht umsonst, denn die mediale Aufmerksamkeit konzentriert sich auf E-Petitionen, die es über diese Hürde schaffen.

Für diese Arbeit wurden die 886 E-Petitionen untersucht, die zwischen dem 14. Oktober 2008 und dem 19. Januar 2010 eingingen und während ihrer Mitzeichnungsfrist mindestens einen Unterstützer gefunden hatten. 495.611 Nutzer zeichneten während dieses Zeitraums mindestens eine E-Petition. Die Summe aller Mitzeichnungen beträgt 1.099.541. Nur etwa 50 E-Petitionen konnten mehr als 50.000 Mitzeichner gewinnen. Die Zahl der E-Petitionen, die dies innerhalb der ersten drei Wochen erreichten, und deren Petenten dadurch in öffentlicher Sitzung des Petitionsausschuss gehört wurden, ist noch deutlich kleiner. Dennoch richtet sich die Medienaufmerksamkeit fast ausschließlich auf

25 Vgl. http://epetitionen.bundestag.de.

26 Für eine detaillierte Diskussion siehe RalfLindner / Ulrich Riehm, a.a.O. (Fn. 18).

27 In diesem Artikel gebrauchen wir den Begriff „E-Petitionen“ synonym zu „Öffentlichen Petitionen“ in Abgrenzung zu „Onlinepetitionen“.

28 Für eine ausführliche Diskussion des formalen Verfahrens siehe Ralf Lindner / Ulrich Riehm, a.a.O. (Fn. 18), S. $503-506$.

29 Thomas Vitzhum, a.a.O. (Fn. 4).

30 Heike Dietrich / Sonja Haase, Berlinerin sagt Ursula von der Leyen die Meinung, in: Berliner Morgenpost vom 10. Mai 2009, http://www.morgenpost.de/berlin/article1088836/Berlinerin_ sagt_Ursula_von_der_Leyen_die_Meinung.html (Abruf am 21. Februar 2011).

31 Markus Beckedahl, 72 Stunden Countdown für Zensursula-ePetition, in: netzpolitik.org vom 24. Mai 2009, http://www.netzpolitik.org/2009/72-stunden-countdown-fuer-zensursula-epetition/ (Abruf am 21. Februar 2011).

32 Vgl. exemplarisch zu Netzsperren: ebenda; zu Hebammen: Edith Wolber, 5. Mai - Internationaler Hebammentag, Deutscher Hebammen Verband e.V., http://www.hebammenverband.de/index. php?id=764\&tx_ttnews[pointer] $=8 \&$ tx_ttnews[tt_news] $=35 \& \mathrm{tx} \_t$ tnews $[$ backPid] $=8 \& \mathrm{cHash}=\mathrm{c} 5$ 9a9f84811867f277e1097d738d3903 (Abruf am 21. Februar 2011); zum Atomkompromiss: Bündnis 90/Die Grünen, a.a.O. (Fn. 15). 


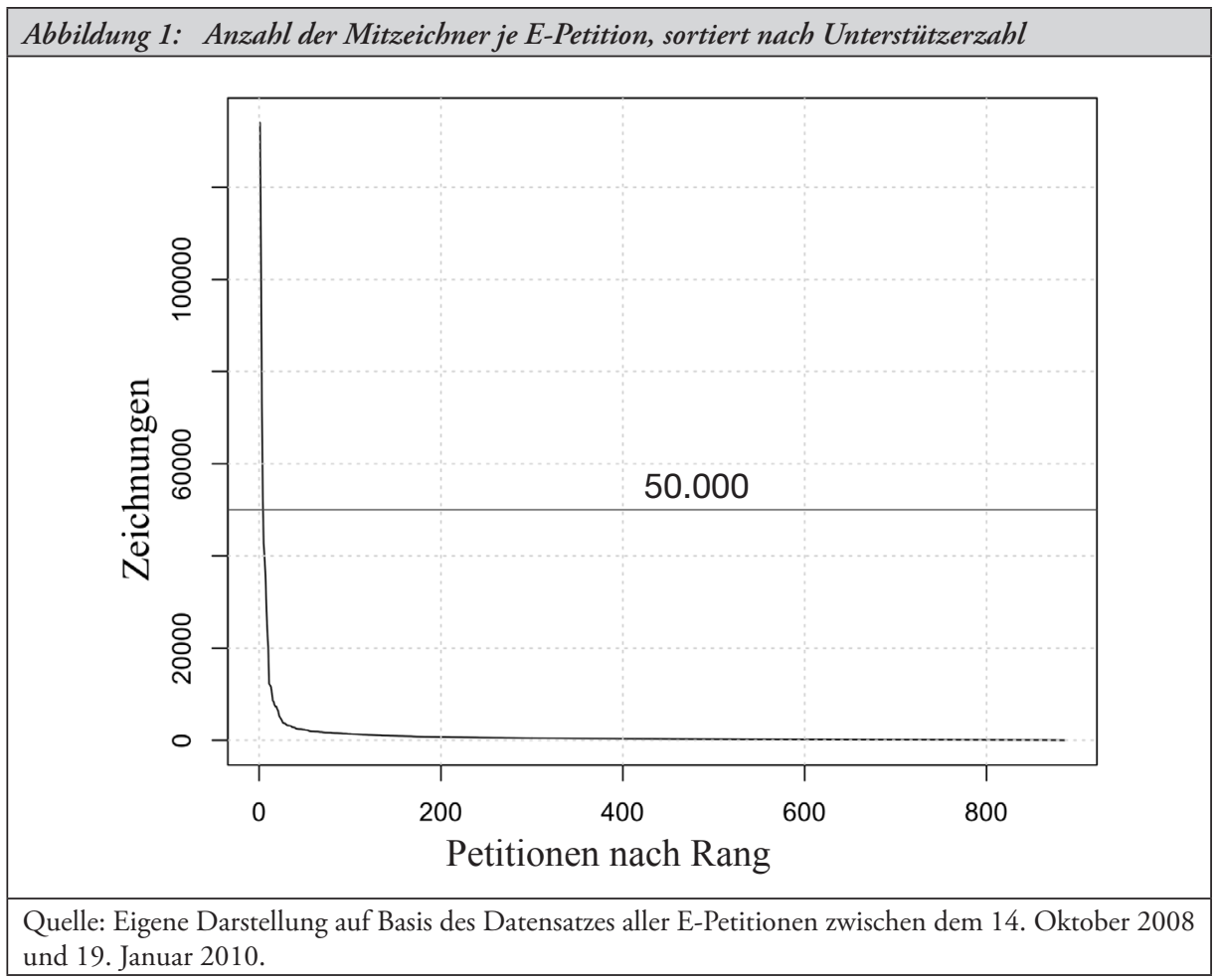

diese wenigen Petitionen. Die ursprüngliche Funktion des Petitionswesen, als Vorschlagsbeziehungsweise Beschwerdekanal für Bürger ${ }^{33}$, wird so durch quasi-plebiszitäre Züge ergänzt. Mitzeichner drücken also nicht nur ihre Unterstützung für das Anliegen einer E-Petition aus, sondern stimmen mit ihrer Mitzeichnung de facto auch darüber ab, ob diese ins Scheinwerferlicht der Medien gerückt wird.

\section{Die Nutzer des E-Petitionssystems: An ihren Taten sollt ihr sie erkennen}

Dieses Spannungsverhältnis zwischen dem Umgang mit E-Petitionen als appellatives Partizipationselement und der quasi-plebiszitären Nutzung wird umso deutlicher, wenn die Nutzung der Plattform im Detail betrachtet wird. Das E-Petitionssystem des Deutschen Bundestags versieht jede Öffentliche Petition mit einer numerischen ID und dokumentiert bei jeder Mitschrift den Namen des Unterzeichners, das von ihm bei seiner Registrierung im System angegebene Heimat-Bundesland, den Tag der Mitzeichnung und die thematische Kategorie, in die die jeweilige E-Petition fällt. Diese Informationen erlauben die Gruppierung von Nutzern der E-Petitionsplattform basierend auf ihrem Verhalten. Dieses Vorgehen beruht auf der Idee, dass Nutzer, die unterschiedliches Verhalten zeigen, auch

33 Vgl. RalfLindner / Ulrich Riehm, a.a.O. (Fn. 18), S. 497 - 500. 
unterschiedliche Motive für ihre Nutzung der Plattform haben. Wer nur einmal eine EPetition mitzeichnet, wird anders motiviert sein als jemand, der über mehrere Monate hinweg wiederholt verschiedene E-Petitionen unterschreibt.

Die Gruppierung nach den Variablen Anzahl der mitgezeichneten E-Petitionen und Dauer der aktiven Nutzung der E-Petitionsplattform lässt erkennen, dass die meisten Nutzer tatsächlich nur ein oder zwei E-Petitionen mitzeichnen. Es existiert eine kleine Gruppe von Nutzern, die bis zu 500 E-Petitionen auf der Plattform unterstützen. Ein ähnliches Bild zeigt sich bei der Zeitspanne: Die überwiegende Mehrzahl der Nutzer, die mehrere EPetitionen mitzeichneten, taten dies in einem Zeitraum von wenigen Tagen. Nur bei einer kleinen Gruppe von Nutzern erstreckt sich die Aktivität über einen Zeitraum von 200 Tagen. Diese Unterschiede im Nutzungsverhalten lassen auf die verschiedene Bedeutung schließen, die Nutzer der Plattform zuschreiben.

Auf Grundlage dieser Daten werden die Nutzer hier in vier Kategorien eingeteilt: (1) Neue Lobbyisten, (2) Hit-and-Run Aktivisten, (3) Aktivismus-Konsumenten und (4) Stakeholder. Diese Gruppen unterscheiden sich danach, ob Nutzer viele E-Petitionen mitzeichneten oder wenige, ob dies in kurzem Abstand geschah oder zwischen ihren Mitzeichnungen längere Zeit verstrich, und ob die verschiedenen von einem Nutzer gezeichneten E-Petitionen in denselben thematischen Kategorien lagen (siehe Abbildung 2).

Unter einem „Neuen Lobbyisten“ verstehen wir einen Nutzer des E-Petitionssystems, der über einen langen Zeitraum (in diesem Fall länger als drei Wochen ${ }^{34}$ ) mindestens drei E-Petitionen mitgezeichnet hat, die in wenige thematische Kategorien fallen. Dies könnte zum Beispiel ein Nutzer sein, der im Januar 2009 eine E-Petition aus der Kategorie „Steuern" unterstützt, dann im Juni eine E-Petition aus derselben Kategorie unterschrieb und im Herbst noch eine E-Petition aus der Kategorie „Schuldrecht“ zeichnete. Dieses Verhalten spricht für Nutzer, die an einem spezifischen Thema interessiert sind und die E-Petitionsplattform als nützliches Instrument der Partizipation gewählt haben. In den hier erfassten Daten fanden sich 269 Personen, die dieses Verhalten zeigten.

Ein „Hit-and-Run Aktivist“ ist definiert als ein Nutzer, der verschiedene E-Petitionen aus wenigen thematischen Kategorien gezeichnet hat. Er unterscheidet sich vom „Neuen Lobbyisten" dadurch, dass er sich diesen E-Petitionen nur während eines kurzen Zeitraums (in diesem Fall weniger als drei Wochen) angeschlossen hat. Es könnte zum Beispiel ein Nutzer sein, der am 12. Juni 2009 eine E-Petition aus der Kategorie „Internet“ mitzeichnete, am selben Tag eine weitere aus dieser Kategorie einen Tag später eine E-Petition aus der Kategorie „Schuldrecht“ unterschrieb, ohne jedoch danach noch einmal die E-Petitionsplattform wiederzuverwenden. Dieses Verhalten deutet auf Nutzer, die auf die Plattform aufmerksam gemacht wurden (sei es zum Beispiel durch eine Kampagnenveranstaltung, einen Nachrichtenbeitrag oder durch Nachrichten in einer Sozialen Netzwerkplattform) und einige E-Petitionen ihres Interesses fanden. Nachdem sie einige davon mitgezeichnet hatten, kehrten diese Nutzer allerdings nicht wieder zu der Plattform zurück. Dieser Typus zeigt also kein langfristiges Interesse, die E-Petitionsplattform als Kanal der politischen Partizipation zu nutzen.

34 Dieser Zeitraum wurde gewählt, weil er der medialen Halbwertszeit von Petitionen entspricht diese haben nur drei Wochen, innerhalb derer sie eine Anhörung vor dem Ausschuss erreichen können (siehe oben). 


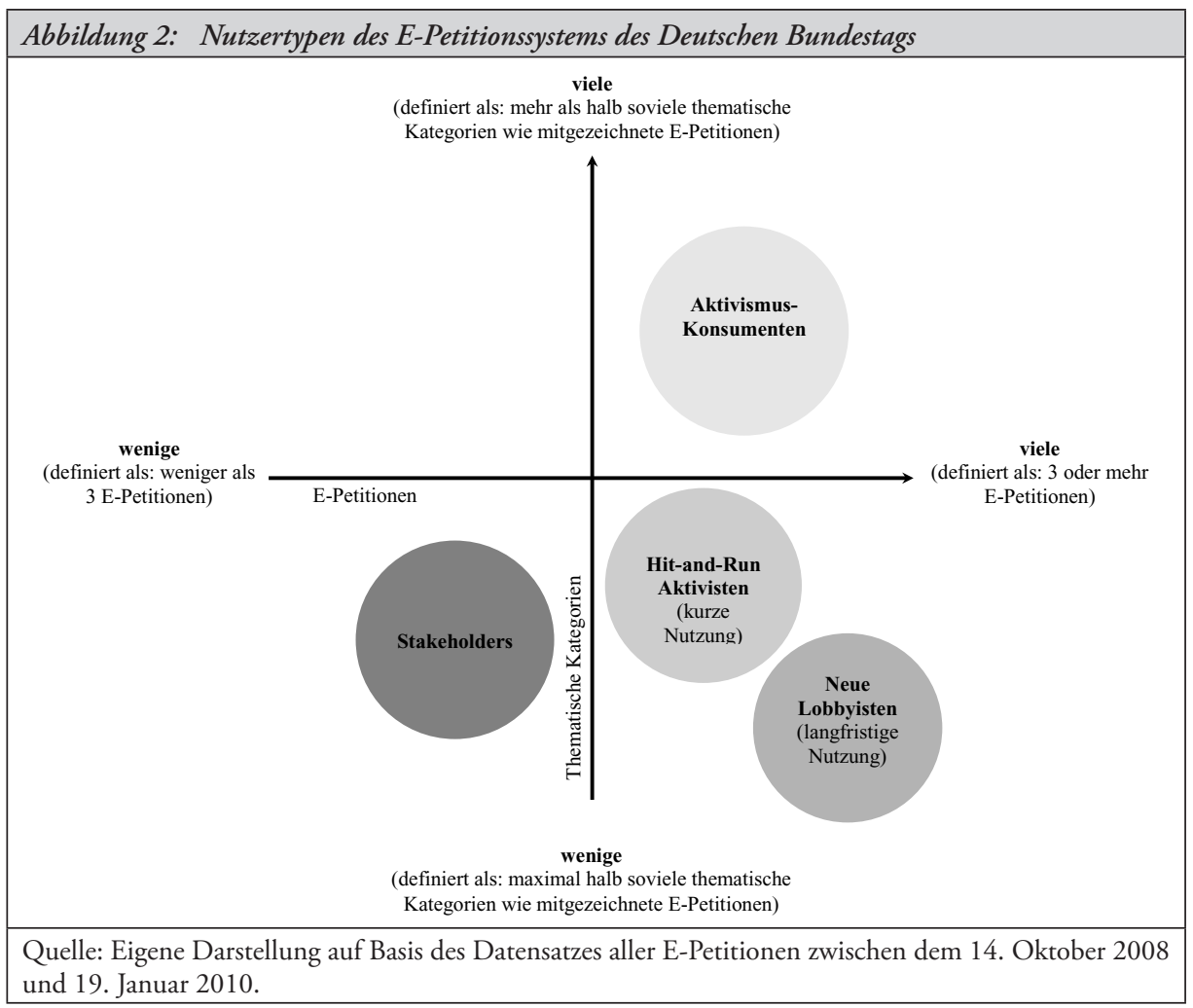

Die „Aktivismus-Konsumenten“ sind Nutzer, die mehr als drei E-Petitionen aus unterschiedlichen thematischen Kategorien zeichneten. Der Zeitraum spielt bei der Kategorisierung dieses Nutzertyps keine Rolle. Es könnte zum Beispiel jemand sein, der im Januar 2009 eine E-Petition aus der Kategorie „Steuern“, im Juni eine aus der Kategorie „Internet“ und im Herbst noch eine E-Petition aus dem „Schuldrecht" mitzeichnete. Diese Nutzer haben kein besonderes langfristiges Interesse an spezifischen politischen Themen, unterschreiben aber die E-Petitionen um des Mitzeichnens willen. 80.278 Nutzer zeigten dieses Verhalten.

Als „Stakeholder“ wird hier ein Nutzer definiert, der auf der Plattform nur ein oder zwei E-Petitionen gezeichnet hat. Dies könnte zum Beispiel jemand sein, der im Juni 2009 eine E-Petition aus der Kategorie „Steuern“ mitzeichnete und im September 2009 eine weitere aus derselben Kategorie unterschrieb. Außerdem fallen alle Nutzer in diese Kategorie, die nur eine E-Petition mitzeichneten. Dieser Typ entspricht dem „klassischen Petenten“, der an einem spezifischen Thema interessiert ist und versucht, es durch Petitionen an die dafür zuständigen Institutionen anzusprechen.

Tabelle 1 zeigt, dass die meisten Petenten die Plattform nutzen, um bloß ein oder zwei E-Petitionen mitzuzeichnen. Dies sind die „Stakeholder“ genannten Nutzer. Eine weitere große Gruppe stellen die „Aktivismus-Konsumenten“ dar, die das E-Petitionssystem nur während einer kurzen Zeitspanne nutzen, um zahlreiche, thematisch nicht verwandte EPetitionen mitzuzeichnen. 


\begin{tabular}{|l|c|}
\hline \multicolumn{2}{|l|}{ Tabelle 1: Nutzertypen und ihre Häufigkeit } \\
\hline Typus & $\mathrm{N}$ \\
\hline Neue Lobbyisten & 269 \\
Hit-and-Run Aktivisten & 235 \\
Aktivismus-Konsumenten & 80.278 \\
Stakeholder & 414.829 \\
\hline Quelle: Eigene Darstellung auf Basis des Datensatzes aller E-Petitionen zwischen dem 14. Oktober 2008 \\
und 19. Januar 2010. \\
\hline
\end{tabular}

\section{Der Mitzeichner-Overspill}

Bei den in Abbildung 1 gezeigten großen Unterschieden in den Unterstützerzahlen von EPetitionen an den Bundestag fällt als ein Extrem auf, dass einige wenige E-Petitionen die überwiegende Anzahl der Mitzeichnungen auf sich vereinigen konnten. In unserem Untersuchungszeitraum konnten nur vierzehn E-Petitionen mehr als 10.000 Unterstützer gewinnen. Diese vierzehn Eingaben, also deutlich unter zwei Prozent der 886 auf der Plattform veröffentlichten, bündeln in der Summe allerdings gut die Hälfte der 1.099.541 Mitzeichnungen, wobei unter ihnen zehn die Schwelle von 50.000 Unterstützern nicht überspringen konnten.

In der Regel gingen auf der E-Petitionsplattform täglich Unterschriften in hohen Hunderter- beziehungsweise niedrigen Tausenderzahlen ein. Diese Unterschriften konzentrierten sich auf E-Petitionen, die im Verlauf ihrer Mitzeichnungsfrist weniger als $10.000 \mathrm{Un}$ terschriften sammeln konnten. Dieser Trend wurde während des Untersuchungszeitraums mehrmals gebrochen. An einzelnen Tagen waren auf der E-Petitionsplattform plötzlich weit mehr als 5.000 Unterschriften zu verzeichnen. Diese plötzlichen Veränderungen in der Nutzung wurden von einigen wenigen E-Petitionen verursacht, die über 10.000 Unterschriften sammeln konnten.

Bei genauerer Betrachtung des Zeitverlaufs fällt eine weitere Regelmäßigkeit auf. Zu Beginn des Untersuchungszeitraums erhalten die E-Petitionen mit insgesamt weniger als 10.000 Mitzeichnungen täglich in der Summe einige hundert Unterschriften. Dies ändert sich jedoch auffällig, sobald die E-Petition für das bedingungslose Grundeinkommen hohe Mitzeichnerzahlen anzuziehen beginnt. Solange diese erfolgreiche E-Petition aktiv ist, erhalten auch Eingaben mit niedrigeren Unterstützerzahlen zusammen mehr Unterschriften als zuvor. Der Erfolg einer E-Petition scheint also, etwas abgeschwächt, auch auf andere, in dem Zeitraum aktive E-Petitionen überzuspringen. Es kommt also zu einem MitzeichnerOverspill. Dieses Muster tritt auch im weiteren Verlauf auf. Handelt es sich hierbei um einen Zufall, oder besteht ein statistischer Zusammenhang?

Unter Zuhilfenahme eines häufig gebrauchten Tests für die statistische Abhängigkeit von Zeitreihen ${ }^{35}$ lässt sich ein regelmäßiger Zusammenhang zwischen der Entwicklung der

35 Durch die Verwendung des R Pakets „vars“ [dokumentiert in: Bernhard Pfaff, VAR, SVAR and SVEC Models: Implementation, within R Package vars, in: Journal of Statistical Software, 27. Jg. (2008), H. 4, S. 1 - 32], wurde die F-Type Causality getestet [eingeführt in: Clive W. J. Granger, Investigating Causal Relations by Econometric Models and Cross-spectral Methods, in: Econometrica, 37. Jg. (1969), H. 3, S. 424 - 238]. Seit Christopher A. Sims [Macroeconomics and 


\begin{tabular}{|c|c|c|c|}
\hline \multicolumn{4}{|c|}{ Tabelle 2: E-Petitionen mit über 10.000 Mitzeichnern } \\
\hline ID & $\begin{array}{l}\text { Thematische Kategorie auf } \\
\text { der E-Petitionsplattform }\end{array}$ & Bezeichnung & $\begin{array}{c}\text { Zahl der } \\
\text { Mitzeichner }\end{array}$ \\
\hline 3.860 & Internet & Keine Indizierung und Sperrung von Webseiten & 134.015 \\
\hline 4.517 & Bürgerliches Recht & $\begin{array}{l}\text { Gesellschaft für musikalische Aufführungs- und } \\
\text { mechanische Vervielfältigungsrechte (GEMA) }\end{array}$ & 106.575 \\
\hline 4.958 & $\begin{array}{l}\text { Straftaten gegen die } \\
\text { öffentliche Ordnung }\end{array}$ & Gegen ein Verbot von Action-Computerspielen & 73.002 \\
\hline 1.422 & $\begin{array}{l}\text { Reformvorschläge in der } \\
\text { Sozialversicherungen }\end{array}$ & Bedingungsloses Grundeinkommen & 52.973 \\
\hline 5.178 & Hochschulwesen & Masterstudienplatz für Bachelorabsolventen & 42.740 \\
\hline 8.236 & Steuerpolitik & Einführung einer Finanztransaktionsteuer & 39.565 \\
\hline 4.145 & Waffenrecht & Gegen ein Verbot von Spielen z.B. Paintball & 35.827 \\
\hline 4.525 & $\begin{array}{l}\text { Bodenverwertungs- und } \\
\text {-verwaltungs GmbH }\end{array}$ & $\begin{array}{l}\text { Verzicht der weiteren Privatisierung von } \\
\text { Gewässern }\end{array}$ & 28.612 \\
\hline 7.922 & $\begin{array}{l}\text { Wissenschaft und } \\
\text { Forschung }\end{array}$ & $\begin{array}{l}\text { Kostenloser Erwerb wissenschaftlicher } \\
\text { Publikationen }\end{array}$ & 23.631 \\
\hline 8.308 & Schuldrecht & $\begin{array}{l}\text { Kostenfreiheit bei fristgerechter Beseitigung } \\
\text { des Abmahngrundes }\end{array}$ & 20.113 \\
\hline 3.827 & Bürgerliches Recht & $\begin{array}{l}\text { Offenlegung der Abrechnungsmethoden der } \\
\text { Gesellschaft für musikalische Aufführungs- und } \\
\text { mechanische Vervielfältigungsrechte (GEMA) }\end{array}$ & 12.322 \\
\hline 4.006 & Urheberrecht & Kopierschutzmaßnahmen & 11.931 \\
\hline 4.724 & Einkommensteuer & Häusliches Arbeitszimmer & 11.646 \\
\hline 1.471 & Heilberufe & Vergütung der Ausbildungskandidaten & 10.244 \\
\hline
\end{tabular}

Zahl der Mitzeichnungen für E-Petitionen mit in der Summe mehr als 10.000 Unterschriften und der Entwicklung der Mitzeichnungen für E-Petitionen, die in der Summe unter 10.000 Unterschriften blieben, zeigen: Ein Anstieg der Mitzeichnungen in großen Petitionen führt mit einer Verzögerung von drei Tagen zu einem Anstieg von Mitzeichnungen in kleinen Petitionen. ${ }^{36}$ Der Mitzeichner-Overspill lässt sich also auch durch einen statistischen Zusammenhang belegen. In Zeiten, in denen erfolgreiche E-Petitionen viele Mitzeichner anziehen, steigt also auch die Zahl der Unterschriften für in der Summe weniger erfolgreiche Eingaben. Politische Partizipation führt also zu mehr politischer Partizipation.

Reality, in: Econometrica, 48. Jg. (1980), H. 1, S. 1 - 48] wird dieser Wert häufig in ökonometrischen Modellen genutzt.

36 Für eine ausführliche Dokumentation der Analyse siehe Andreas Jungherr / Pascal Jürgens, a.a.O. (Fn. 23). 


\section{Kleine Teile, lose verknüpft}

„Small pieces loosely joined“ 37 - so David Weinberger über ein Ordnungsprinzip des Internets. Er beschreibt damit eine neuartige Kategorisierung von digitalen Inhalten durch Links, die sich von der ursprünglichen Kategorisierung durch die Urheber der Inhalte löst. Die Nutzungsdaten der Plattform bieten eine Gruppierungsmöglichkeit auf einem ähnlichen Ordnungsprinzip: den Nutzern, die verschiedene E-Petitionen mitzeichneten.

Die automatische Einsortierung einer E-Petition auf der Plattform des Bundestages unter Oberbegriffe (zum Beispiel „Bürgerliches Recht“, „Urheberrecht“ oder „Einkommensteuer") führt zwar dazu, dass man E-Petitionen leicht einer thematischen Gruppe zuordnen kann, jedoch sagen diese Kategorien wenig über die tatsächlichen Nutzungsgewohnheiten der Petenten und Mitzeichner. Bereits ein erster Blick auf die vierzehn E-Petitionen, die über 10.000 Mitzeichner gewinnen konnten, und den Themenbereich dem sie auf der E-Petitionsplattform zugeordnet wurden, zeigt, dass die erfolgreichsten Eingaben in unterschiedliche Kategorien fallen. Verließe man sich nur auf diese, so würde man wenige Gemeinsamkeiten zwischen den populären E-Petitionen finden.

Tabelle 3 zeigt exemplarisch an den drei E-Petitionen, die im Untersuchungszeitraum die meisten Unterschriften sammeln konnten, dass die erfolgreichen Eingaben einen großen gemeinsamen Stamm an Mitzeichnern haben. So teilt zum Beispiel die dritterfolgreichste E-Petition - \#4.958 „Straftaten gegen die öffentliche Ordnung” - mit insgesamt 73.002 Mitzeichnern 35 Prozent dieser Mitzeichner mit der E-Petition \#3.860 „Keine Indizierung und Sperrung von Webseiten”. Knapp 20 Prozent ihrer Mitzeichner unterschrieben zusätzlich die zweiterfolgreichste E-Petition \#4.517 „Gesellschaft für musikalische Aufführungs- und mechanische Vervielfältigungsrechte (GEMA)”. Dies deutet auf systematische Muster in der Unterstützung unterschiedlicher E-Petitionen hin. Auch wenn diese drei Eingaben in verschiedene thematische Gruppen der E-Petitionsplattform fallen (in diesem Fall die Kategorien „Internet“, „Bürgerliches Recht“ und „Straftaten gegen die öffentliche Ordnung"), fühlt sich ein Großteil ihrer Mitzeichner von allen drei angespro-

\begin{tabular}{|c|c|c|c|}
\hline \multicolumn{4}{|c|}{ Tabelle 3: Anzahl der Mitzeichner, die zwei der erfolgreichsten drei E-Petitionen unterschrieben } \\
\hline & \#3.860 & \#4.517 & \#4.958 \\
\hline $\begin{array}{l}\text { \#3.860 „Keine Indizierung und Sperrung von } \\
\text { Webseiten” }\end{array}$ & - & $\begin{array}{c}19,127 \\
(14,27 \% \text { der } \\
\text { Zeichner von } \\
\# 3.860)\end{array}$ & $\begin{array}{l}25,565 \\
(19,08 \% \text { der } \\
\text { Zeichner von } \\
\# 3.860)\end{array}$ \\
\hline $\begin{array}{l}\text { \#4.517 „Gesellschaft für musikalische Auffüh- } \\
\text { rungs- und mechanische Vervielfältigungsrech- } \\
\text { te (GEMA)” }\end{array}$ & $\begin{array}{l}19,127 \\
(17,27 \% \text { der } \\
\text { Zeichner von } \\
\# 4.517)\end{array}$ & - & $\begin{array}{l}14,582 \\
(13,68 \% \text { der } \\
\text { Zeichner von } \\
\# 4.517)\end{array}$ \\
\hline $\begin{array}{l}\text { \#4.958, „Straftaten gegen die öffentliche Ord- } \\
\text { nung” }\end{array}$ & $\begin{array}{c}25,565 \\
(35,02 \% \text { der } \\
\text { Zeichner von } \\
\# 4.958)\end{array}$ & $\begin{array}{c}14,582 \\
(19,97 \% \text { der } \\
\text { Zeichner von } \\
\# 4.958)\end{array}$ & - \\
\hline
\end{tabular}

37 David Weinberger, Small Pieces Loosely Joined: A Unified Theory of the Web, Jackson 2002. 


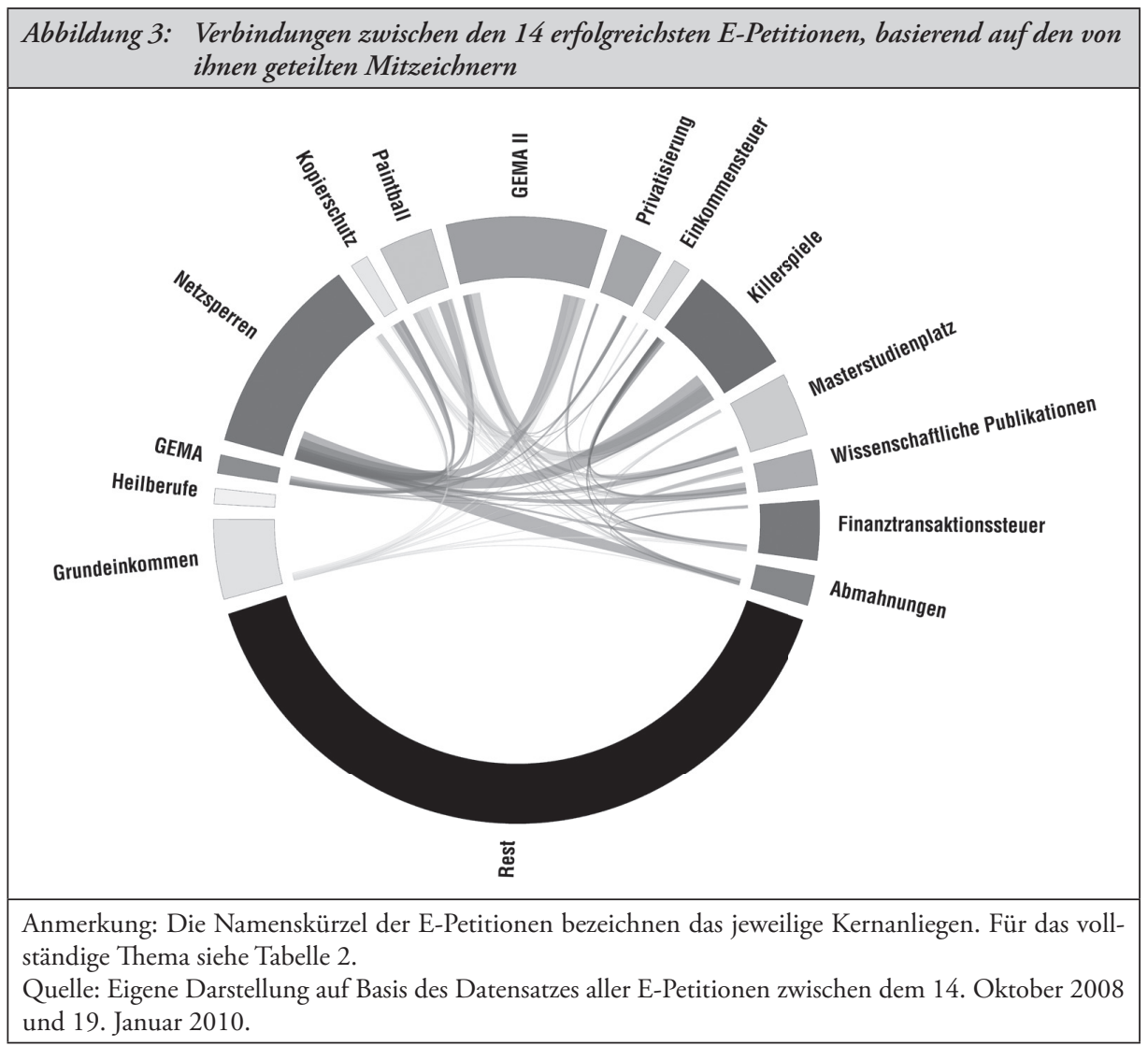

chen. Die Zeichnung verschiedener E-Petitionen ermöglicht Rückschlüsse auf deren Verknüpfungen in der Wahrnehmung der Plattformnutzer.

In Abbildung 3 werden die Verbindungen durch gemeinsame Mitzeichner zwischen den 14 populärsten E-Petitionen dargestellt. Diese sind im Uhrzeigersinn, in der zeitlichen Reihenfolge ihrer Veröffentlichung, sortiert. Die Darstellung beginnt links außen mit der ersten, die mehr als 10.000 Unterschriften sammeln konnte: \#1.422 „Bedingungsloses Grundeinkommen“. Die Linien zwischen den E-Petitionen zeigen gemeinsame Mitzeichner. Je breiter die Linien zwischen zwei E-Petitionen, desto mehr Mitzeichner teilen diese.

Das Schaubild zeigt, dass viele Unterstützer der E-Petition \#1.422 „Bedingungsloses Grundeinkommen“ das E-Petitionssystem auch weiterhin häufig nutzten. Strahlenförmig gehen Verbindungen von dieser Eingabe zu verschiedenen der darauffolgenden aus, nicht zuletzt zur \#3.860 „Keine Indizierung und Sperrung von Webseiten“, die im Untersuchungszeitraum die erfolgreichste E-Petition war. Bei dieser zeigt sich ein ähnliches Bild. Es existieren klare Verbindungen zu den E-Petitionen \#4.006 „Kopierschutzmaßnahmen“, \#4.145 „Gegen ein Verbot von Spielen z.B. Paintball“, \#4.517 „Gesellschaft für musikalische Aufführungs- und mechanische Vervielfältigungsrechte (GEMA)“, \#4.958 „Gegen ein Verbot von Action-Computerspielen“ und \#8.308 „Kostenfreiheit bei fristgerechter Beseitigung des Abmahngrundes“. 
Eine Kategorisierung von E-Petitionen erweist sich als einfach, wenn diese auf Basis von gemeinsamen Mitzeichnern erfolgt. Wie schon die drei erfolgreichsten E-Petitionen fallen auch die Eingaben dieser Gruppe in ganz unterschiedliche thematische Kategorien der Plattform. Dennoch sind die zahlreichen gemeinsamen Mitzeichner ein Indiz für zugrunde liegende Gemeinsamkeiten. In diesem Fall lässt sich die Gemeinsamkeit leicht identifizieren. Alle E-Petitionen dieser Kategorie betreffen Anliegen einer netzaktiven Bevölkerungsgruppe: Netzsperren, Kopierschutzmaßnahmen, Paintball, GEMA-Gebühren, Killerspiele und kostenpflichtige anwaltliche Abmahnungen fallen eindeutig in die Lebensrealität junger, netzaffiner Bürger. ${ }^{38}$

Die systematische Untersuchung von Mitzeichnungsmustern erlaubt also eine bisher nicht zugängliche Einteilung von E-Petitionen und politischen Themen nach ihrer Zusammengehörigkeit in der Lebensrealität der Plattformnutzer. ${ }^{39}$

\section{Tausendmal geklickt, tausendmal ist nichts passiert}

Die überwiegende Zahl der öffentlichen E-Petitionen, die auf der E-Petitionsplattform des Deutschen Bundestags veröffentlicht werden, werden nur von wenigen Nutzern mitgezeichnet. Diese Eingaben werden zwar durch den Petitionsausschuss geprüft und bearbeitet, die mediale Aufmerksamkeit richtet sich allerdings fast ausschließlich auf die E-Petitionen, die über die „magische Grenze “ 40 von 50.000 Unterschriften springen. Dies mag kein ganz ungewollter Nebeneffekt der Einführung der E-Petitionsplattform sein. In einem der drei Anträge der Fraktionen SPD und Bündnis 90/Die Grünen im Petitionsausschuss, auf denen die Einführung der E-Petitionsplattform basiert, ist zu lesen: „Es erscheint daher angemessen, Petitionen, hinter denen eine massenhafte Anzahl von Petentinnen und Petenten steht, anders wahrzunehmen als Einzelpetitionen mit Individualanliegen. Während letztere unbedingt Diskretion und Datenschutz bei der Behandlung ihrer Petition gewahrt haben wollen, geht es den Unterzeichnerinnen und Unterzeichnern von Massen- und Sammelpetitionen gerade um die Herstellung von Öffentlichkeit und das Hinzugewinnen von Unterstützerinnen und Unterstützern. “41 Erklärend wird ergänzt: „Durch die beantragten Änderungen soll - wie dargelegt - der Charakter des Petitionsrechts als politisches Mitwirkungsrecht stärker zur Geltung kommen. Dies soll durch eine stärkere Beachtung bestimm-

38 Hier soll jedoch nicht der Eindruck erweckt werden, dass nur junge, netzaffine Bevölkerungsteile die E-Petitionsplattform nutzen. Der große Erfolg anderer, netzunabhängiger E-Petitionen spricht für die Attraktivität der Plattform auch für Bevölkerungsgruppen mit geringerem Interesse an Internetthemen (siehe zum Beispiel \#1.422 „Bedingungsloses Grundeinkommen“, \#8.236 „Einführung einer Finanztransaktionsteuer“, \#4.525 „Verzicht der weiteren Privatisierung von Gewässern“" oder \#4.724 „Häusliches Arbeitszimmer").

39 Für eine ausführlichere quantitative Analyse hierzu siehe Andreas Jungherr / Pascal Jürgens, a.a.O. (Fn. 23).

40 Thomas Vitzhum, a.a.O. (Fn. 4).

41 Antrag der Fraktionen SPD und Bündnis 90/Die Grünen zur Änderung der Grundsätze des Petitionsausschusses über die Behandlung von Bitten und Beschwerden (Verfahrensgrundsätze) Öffentliche Anhörung von Petenten bei Massen- und Sammelpetitionen, Petitionsausschuss, Ausschussdrucksache $15 / 151$ vom 23. November 2004, S. 1. 
ter Sammel- und Massenpetitionen im parlamentarischen Willensbildungsprozess unter Einbeziehung der Öffentlichkeit geschehen. “42

Dabei ist anzumerken, dass die oben beschriebenen Entwicklungen, also die Konzentration der medialen Aufmerksamkeit auf einige wenige E-Petitionen und die zunehmende „Horse-Race“-Berichterstattung, bereits in der Gestaltung der E-Petitionsplattform angelegt waren. Hier liegt aber auch die Ursache für ein grundlegendes Problem der E-Petitionsplattform. Das in Art. 17 GG formulierte Petitionsrecht ermöglicht es Bürgern zwar, sich mit ihren Anliegen an die entsprechenden staatlichen Akteure zu wenden; eine automatische Durchsetzung ihrer Interessen ist jedoch mit diesem Recht nicht verbunden. In Deutschland soll der Petitionsausschuss des Deutschen Bundestags Petitionen „nicht nur entgegennehmen, sondern auch wahrnehmen, prüfen, über die Begründetheit der Petition entscheiden und dem Petenten darüber eine Mitteilung („Bescheid“) zugehen lassen“43. Auch wenn dies im internationalen Vergleich eine starke Selbstverpflichtung der staatlichen Institutionen ist, entspricht es wohl kaum dem „Mitwirkungsrecht“, das den Fraktionen von SPD und Bündnis 90/Die Grünen bei ihren Anträgen zur Modernisierung des Petitionsrechts vorschwebte. ${ }^{44}$ Auch die inzwischen eingeführte „Öffentliche Anhörung von Petenten von Massen- und Sammelpetitionen" stellt sich als nicht ausreichend heraus, um die plötzlich geweckten Mitbestimmungsansprüche der Einreicher und Mitzeichner von erfolgreichen E-Petitionen zu befriedigen. ${ }^{45}$ Diese Auffassung vertreten auch Fürsprecher zusätzlicher direktdemokratischer Instrumente im deutschen Gesetzgebungsprozess. Hier erscheinen Sammel- und Massenpetitionen als ein erster Schritt zu mehr direktdemokratischen Gestaltungsmöglichkeiten. ${ }^{46}$ E-Petitionen mit hohen Mitzeichnerzahlen werden also als Quasi-Plebiszite interpretiert und kommuniziert, ohne jedoch die tatsächliche gesetzliche Verbindlichkeit von Volksentscheiden zu besitzen.

Darin liegt ein potentieller Konflikt. Petenten werden durch die E-Petitionsplattform des Deutschen Bundestags ermutigt, durch schnelle Kampagnen viele Unterstützer für ihre Anliegen zu sammeln. Damit besteht die Möglichkeit rascher Öffentlichkeit für einige wenige, populäre E-Petitionen und ihrer Initiatoren. Auf Seite der Unterstützer wird die Erwartung geweckt, dass durch ihre Mitzeichnung tatsächlich politische Vorhaben in der von ihnen gewünschten Weise beeinflusst werden. Wenn dann allerdings das einzige verbindliche Resultat dieser spontanen Mobilisierung eine öffentliche Anhörung vor dem Petitionsausschuss ist, besteht die Gefahr, dass die Unterzeichner massiv enttäuscht werden. Als In-

42 Ebenda, S. 3.

43 Ulrich Riehm / Christopher Coenen / RalfLindner / Clemens Blümel, a.a.O. (Fn. 20), S. 39.

44 Antrag der Fraktionen SPD und Bündnis 90/Die Grünen zur Änderung der Grundsätze des Petitionsausschusses über die Behandlung von Bitten und Beschwerden (Verfahrensgrundsätze) Ermöglichung von E-Mail Petitionen, Petitionsausschuss, Ausschussdrucksache 15/149 vom 23. November 2004; Antrag der Fraktionen SPD und Bündnis 90/Die Grünen zur Änderung der Grundsätze des Petitionsausschusses über die Behandlung von Bitten und Beschwerden (Verfahrensgrundsätze) Modellversuch zur Mitzeichnung von Petitionen im Internet, Petitionsausschuss, Ausschussdrucksache 15/150 vom 23. November 2004; Ausschussdrucksache 15/151, a.a.O. (Fn. 41).

45 Vgl. Steffen Kraft, Wir bereiten uns vor, in: Der Freitag vom 17. Juni 2009, http://www.freitag. de/alltag/0925-franziska-heine-interview-verfassungsklage-zensur (Abruf am 6. März 2011).

46 Vgl. Wolfgang Ismayr, Vitalisierung der parlamentarischen Demokratie durch Petitionen?, in: Reinhard Bockhofer (Hrsg.), Demokratie wagen - Petitionsrecht ändern!, Bremen 2004, S. 60 73. 
diz hierfür lässt sich die extrem niedrige Zahl der Plattformnutzer lesen, die nur ein oder zwei E-Petitionen mitzeichneten und danach die Plattform nicht mehr verwendeten.

Wenn also der „Aufstand der Netzbürger“ 47 auf das gelassene „wir sollten vielleicht chatten" 48 der gewählten Volksvertreter trifft, ist unklar, welche gestalterische Form der vorher geweckte Mitbestimmungsanspruch erhält. Vielleicht steht exemplarisch für dieses Gefühl eine Äußerung des Twitter-Nutzers Max Winde: „Ihr werdet euch noch wünschen, wir wären politikverdrossen." ${ }^{49}$

47 Marcel Rosenbach / Hilmar Schmundt, Aufstand der Netzbürger, in: Der Spiegel vom 3. August 2009, S. $26-28$.

48 Ole Reißmann, Vielleicht sollten wir chatten, in: Spiegel Online vom 22. Februar 2010, http:// www.spiegel.de/netzwelt/netzpolitik/0,1518,679582,00.html (Abruf am 18. Februar 2011).

49 MaxWinde (@343max), Ihr werdet euch noch wünschen wir wären politikverdrossen, 18. Juni 2009, http://twitter.com/343max/status/2228357957 (Abruf am 18. Februar 2011).

\title{
Noch immer „Stiefkind der Sozialwissenschaften“? Ein Plädoyer für mehr politikwissenschaftliche Forschung über Rechnungshöfe*
}

\author{
Markus Seyfried
}

Es gibt nur wenige Institutionen, die von den Sozialwissenschaften so konsequent ignoriert werden wie die Rechnungshöfe. Dies gilt im deutschen Fall umso mehr für die Landesrechnungshöfe. Auf den ersten Blick mag dies verwundern, da die Schuldenlast der öffentlichen Haushalte immer mehr zunimmt und die Bedeutung der Finanzkontrolleure als kritische „Generalisten"1 steigen müsste. Auf den zweiten Blick jedoch spiegelt sich in dem wissenschaftlichen Desinteresse das generelle Schattendasein wider, das die Rechnungshöfe fristen. Die Institutionen der Finanzkontrolle werden vom Bürger weitgehend ignoriert - abgesehen von einigen wenigen medienwirksamen Skandalen - und von der Politik mitunter milde belächelt.

Die geringe Beachtung der Rechnungshöfe - ähnlich wie von Nils Diederich u.a. in Bezug auf den Bundesrechnungshof festgestell $\mathrm{t}^{2}$ - verwundert insofern, als gerade die Sozialwissenschaften dafür prädestiniert sind, sich an schwer greifbare Untersuchungsgegenstän-

* Für sehr hilfreiche Anmerkungen und Kritik danke ich Julia Fleischer, Sylvia Veit und Bastian Jantz sowie der ZParl-Redaktion.

1 Vgl. Werner Jann / Kai Wegrich, Wie bürokratisch ist Deutschland? Und warum? Generalisten und Spezialisten im Entbürokratisierungsspiel, in: Der moderne Staat, 1. Jg. (2008), H. 1, S. 49 - 72; Hans Herbert von Arnim, Gemeinwohl und Gruppeninteressen. Die Durchsetzungsschwäche allgemeiner Interessen in der pluralistischen Demokratie, Frankfurt am Main 1977, S. 373; ders., Wirksamere Finanzkontrolle bei Bund, Ländern und Gemeinden. Analyse und Reformvorschläge, Karl-Bräuer Institut des Bundes der Steuerzahler, H. 42, Bad Wörishofen 1978, S. 54.

2 Vgl. Nils Diederich / Franz-Otto Gilles / Gerhard Otto / Gundolf Otto / Rainer Weinert, Die Institution Rechnungshof, Stiefkind der Sozialwissenschaften, in: ZParl, 15. Jg. (1984), H. 4, S. $479-$ 494, S. 493 f. 